\title{
Cor e Qualidade Formal no Rorschach de Adolescentes e Jovens Adultos
}

\author{
Danilo R. Silva ${ }^{1}$ \\ Academia das Ciências de Lisboa \\ Ana Sousa Ferreira \\ Faculdade de Psicologia, Universidade de Lisboa; BRU - Instituto Universitário de Lisboa
}

\section{RESUMO}

O presente artigo procura verificar se a cor será causa de maior frequência de distorção perceptiva no Rorschach, como o sugerem alguns estudos normativos. A aplicação dos cartões II, III, VIII, IX e X, na sua versão original e numa versão cromática, a dois grupos de jovens de ambos os sexos, permitiu verificar, com respeito às variáveis Fo, F-, Fu, XA, tendo em conta as características cromático/acromático e masculino/feminino, o seguinte: quanto à variável Fo, verifica-se uma diferença estatisticamente significativa, favorável ao sexo feminino, no cartão IX; quanto à variável Fu, ocorre uma diferença estatisticamente significativa, favorável à versão cromática no cartão X, que se repete com a variável XA. A análise de variância a dois fatores, cromático/acromático e sexo, revela que o sexo feminino é responsável pelo seu valor mais elevado no cartão IX, tanto em Fo como em XA. O presente estudo não permite estabelecer uma relação de causalidade entre a variável cor e a distorção perceptiva no Rorschach.

Palavras-chave: Rorschach; cor; forma; distorção perceptiva.

\section{ABSTRACT - Color and Form Quality in the Rorschach of Adolescents and Young Adults}

This article tries to find if color may the cause of higher frequency in some Rorschach normative studies, it was observed that the highest frequency of perceptive distortion, as it is suggested by some normative studies. Administration of cards II, III, VIII, IX and $\mathrm{X}$, in their original and achromatic versions, to two groups of 40 both sex young people, allowed to get the following results: Concerning FQo variable, it occurs a statistically significant difference favoring the female sex in Card IX; Concerning FQ-variable, no statistically significant difference occurs; Concerning FQu, a statistically difference occurs, in card X, favourable to chromatic version it also occurs with XA variable; Analysis of variance to two factors, chromatic/achromatic and sex, reveals that female sex factor is responsible for higher level of FQo and XA in card IX. Current study does not permit to establish a causal relationship between color and perceptive distortion in Rorschach.

Keywords: Rorschach; color; form; perceptive distortion.

\section{RESUMEN - Color y Calidad Formal en el Rorschach de Adolescentes y Jóvenes Adultos}

El presente artículo busca verificar si el color sería la causa más frecuente de distorsión perceptiva, en el Rorschach, tal como lo sugieren algunos estudios normativos. La aplicación de las láminas II, III, VIII, IX y X, en su versión original y en una versión cromática, a dos grupos de 40 jóvenes de ambos sexos, permitió verificar las características cromático/acromático y masculino/femenino con respecto a las variables Fo, $\mathrm{F}-$, Fu, XA. La variable Fo indicó una diferencia estadística significativa, favorable al sexo femenino, en la lámina IX; en lo que se refiere a la variable $\mathrm{F}-$, no ocurren diferencias estadísticas significativas; con respecto a la variable Fu, ocurre una diferencia estadística significativa en la lámina X, favorable a la versión cromática, que se repite en la variable XA. El análisis de la varianza a dos factores, cromático/acromático y sexo, demostró, que el sexo femenino es responsable por su valor más elevado en la lámina IX, tanto en Fo como en XA. En conclusión, el presente estudio no permite establecer una relación de causalidad entre el color y la distorsión perceptiva en el Rorschach.

Palabras clave: Rorschach; color; forma; distorsión perceptiva.

O método de Rorschach assenta num instrumento de avaliação constituído por dez cartões com borrões de tinta, cinco dos quais acromáticos, dois que contêm a cor vermelha e três que são integralmente coloridos. A sua aplicação é individual e consiste na apresentação de cada cartão, devendo o respondente dizer, diante dele, o que poderia ser ou o que lhe parece ser aquele borrão.

Esse método tem sido objeto da nossa investigação há vários anos e, tratando-se de estudos sobre o efeito da cor na produção de respostas, temos utilizado, além da 
versão cromática ou padronizada, uma versão acromática dos cartões. Tais estudos permitiram destacar os seguintes efeitos da cor na produção e na qualidade formal de respostas ao teste dos borrões de tinta de Rorschach, em crianças e jovens portugueses:

1. Tendência para uma redução da produção de respostas dadas aos cartões coloridos VIII, IX e X entre os 5 e os 11/12 anos (Silva \& Ferreira, 2009, 2011);

2. Aumento da produção de respostas aos cartões coloridos, a partir dos 15/16 anos (Silva \& Ferreira, 2014);

3. Elevação da distorção perceptiva (QF-), no Cartão III, aos 11/12 anos (Silva \& Ferreira, 2017);

4. Elevação da boa percepção (QFo), nas respostas dadas ao cartão X (Silva \& Ferreira, 2017).

Este último dado referente ao período etário em que a cor adquire o efeito de aumento da produção de respostas - 15/16 anos - determinou que se repetisse o estudo anterior sobre Cor e Qualidade Formal, pois considerou-se que tal transformação, de natureza desenvolvimentista, poderia ter algum novo efeito na qualidade perceptiva das respostas, tal como se verificou no estudo anterior, indicado nos números 2 e 3 .

Vale a pena esclarecer que a razão inicial que nos levou ao estudo da relação entre Cor e Qualidade Formal, no Rorschach, foi o ter-se verificado que, nos estudos normativos com crianças e adultos efectuados em Portugal (Silva, 2002; Pires, 2007), quatro dos cinco cartões em que a cor está presente figuram entre os cinco cujo valor de QF- ou distorção perceptiva é mais elevado. Esse mesmo aspecto ocorre também em dois outros estudos de idêntica natureza realizados com adultos por Exner e Erdberg (2005), nos Estados Unidos da América do Norte, muito embora os ditos cartões não sejam sempre os mesmos. Esse fato levou-nos a formular a hipótese, segundo a qual, a cor poderia ser causa ou estar na origem da elevada ocorrência de distorções perceptivas nas respostas àqueles cartões. A presente comunicação dá conta dos resultados obtidos com uma amostra de jovens.

\section{Método}

\section{Participantes}

A amostra foi constituída por dois grupos de adolescentes e jovens adultos, com idades compreendidas entre os 15 e os 21 anos (ver Tabela 1), a frequentar escolas secundárias situadas na área da cidade do Funchal (Ilha da Madeira) e arredores. Não se procedeu a nenhuma forma específica de selecção, tendo-se apenas solicitado aos professores que referissem aos alunos a realização deste estudo, para o qual se pediam voluntários. O psicólogo, por sua vez, precedia o exame de uma tomada de contacto com o examinando, durante a qual informava sobre o que se iria fazer e inquiria sobre se aceitava colaborar. Cada grupo foi formado por 40 voluntários, 20 de cada sexo, sem indicadores de perturbação de comportamento, num total de 80 .

Tabela 1

Número e Percentagem de Participantes por Idade (em anos)

\begin{tabular}{lccc}
\hline \multicolumn{1}{c}{ Idades (em anos) } & Participantes & Percentagem & Percentagem acumulada \\
\hline $15 / 16$ anos & 10 & 13 & 13 \\
17 & 48 & 60 & 73 \\
18 & 13 & 16 & 89 \\
$19 / 21$ & 9 & 11 & 100 \\
\hline
\end{tabular}

\section{Instrumentos}

Os cartões do Método d Rorschach que foram objecto deste estudo estão identificados como II e III, onde o vermelho se junta ao componente acromático, e os cartões ditos cromáticos, VIII, IX e X, na sua versão padronizada e numa versão acromática. Fez-se ainda preceder a apresentação desses cartões da do Cartão I, acromático, como modo de proporcionar ao examinando conhecimento do tipo de material e tarefa que lhe era proposta, facilitando-lhe assim a devida adaptação. Esse cartão não foi considerado no apuramento dos resultados.

A um dos grupos foi aplicada a versão acromática e ao outro, a versão padronizada. A codificação das respostas esteve a cargo do primeiro autor. Tendo em vista a sua organização, foi solicitada autorização à Secretaria Geral da Educação/Direcção Regional de Educação da Região Autónoma da Madeira. Essa instituição concedeu a autorização para o desenvolvimento do trabalho e permitiu que a psicóloga designada por aquela instituição entrasse em contacto com as quatro escolas secundárias, providenciando reunião com os respectivos psicólogos. Tivemos, assim, a oportunidade de contactar previamente com os nossos colaboradores a quem apresentámos os objectivos e quantitativos de examinandos. Recomendámos que estes últimos deveriam frequentar os anos de escolaridade correspondentes às suas idades e serem voluntários, para um trabalho de investigação.

As aplicações foram individuais e estiveram a cargo do primeiro autor. Tiveram lugar em locais adequados das quatro escolas secundárias referidas, ao abrigo de 
eventuais interrupções ou outras formas de perturbação. As instruções adoptadas para aplicação do Rorschach foram as propostas por Exner no seu Sistema Integrativo do Rorschach (2003).

\section{Procedimento de Análise de Dados}

$\mathrm{Na}$ análise da Qualidade Formal, consideraram-se quatro variáveis: QFo, QF-, QFu e XA. Elas correspondem sucessivamente à qualidade perceptiva boa, à distorção perceptiva, à qualidade perceptiva boa, mas de rara frequência, e ao total das respostas de qualidade perceptiva boa, isto é, QFo+QFu. Não se considerou o modo de Qualidade Formal QF+, pela sua raridade e ausência de interesse neste estudo.

$\mathrm{Na}$ análise de dados realizada, a cargo do segundo autor, considerou-se inicialmente um estudo correlacional entre as variáveis XA e QF-, em cada cartão e considerando a amostra total, tendo em vista apurar o grau de adequação dos dados globais, considerados os objectivos deste estudo. Dada a distribuição gaussiana das variáveis XA e QF-, utilizou-se no estudo da sua relação, o coeficiente de correlação de Pearson. Seguidamente, aplicou-se um teste de igualdade de valores médios para amostras emparelhadas, relativo àquelas variáveis, em cada cartão e na amostra total, para dispor de informação sobre como se distribuem os respectivos valores observados em cada um dos cartões.

A análise do efeito do carácter acromático e cromático dos Cartões na Qualidade Formal das respostas foi objecto de três estudos em cada uma das variáveis Fo, F-, $\mathrm{Fu}$ e XA: teste de igualdade de valores médios por sexo; teste de igualdade de valores médios por versão acromática e cromática; análise de variância a dois factores (versão acromática /versão cromática e sexo). Em todas as análises inferenciais utilizadas consideraram-se os níveis de significância de $5 \%$ e $1 \%$ e determinou-se, sempre que possível, uma medida da dimensão do efeito.

\section{Resultados}

A Tabela 2 apresenta as medidas descritivas (média e desvio padrão) e os valores do Coeficiente de Correlação de Pearson entre as variáveis XA e QF- na amostra total, para cada um dos cartões aplicados e na junção dos cartões vermelhos II e III e dos cartões coloridos VIII, IX e X.

Tabela 2

Resultados Descritivos (Média e DP) das Variáveis em Estudo e Coeficiente de Correlação de Pearson entre XA e F-e Respectivo Nível de Significância nos Cartões II, III, VIII, IX, X e II+III e VIII+IX+X do Rorschach

\begin{tabular}{|c|c|c|c|c|c|c|}
\hline \multirow{2}{*}{ Cartões } & & \multicolumn{2}{|c|}{$\mathrm{XA}$} & \multicolumn{2}{|c|}{ F- } & \multirow{2}{*}{$\begin{array}{l}\text { Coeficiente } \\
\text { Correlação }\end{array}$} \\
\hline & & Média & $\mathrm{DP}$ & Média & $\mathrm{DP}$ & \\
\hline II & $\mathrm{XA} \& \mathrm{~F}-$ & 0,83 & 1,21 & 0,70 & 0,85 & $-0,25^{*}$ \\
\hline III & $\mathrm{XA} \& \mathrm{~F}-$ & 1,44 & 1,65 & 0,65 & 0,73 & $-0,40^{* *}$ \\
\hline VIII & XA \& F- & 1,09 & 1,48 & 0,69 & 0,85 & $-0,38^{* *}$ \\
\hline IX & $\mathrm{XA} \& \mathrm{~F}-$ & 0,85 & 1,33 & 0,90 & 0,89 & $-0,25^{*}$ \\
\hline $\mathrm{X}$ & XA \& F- & 1,93 & 2,02 & 1,19 & 1,07 & $-0,35^{* *}$ \\
\hline II, III & $X A \& F-$ & 2,26 & 2,14 & 1,35 & 1,16 & $-0,23^{*}$ \\
\hline VIII, IX, X & XA \& F- & 3,86 & 3,21 & 2,78 & 1,93 & $-0,38^{* *}$ \\
\hline
\end{tabular}

Nota. ${ }^{*} p<0.05 ;{ }^{* *} p<0.01$

Olhando para os valores do coeficiente de correlação obtidos e respectivo valor-p, verifica-se que todos são negativos e estatisticamente significativos embora indiquem apenas a existência de relações fracas ou moderada entre as respostas dos participantes no estudo, às variáveis XA e QF-. Por outro lado, observa-se que, nos cartões II e IX, tais valores são menores. Se olharmos para a coluna das médias, verifica-se que os respectivos valores são os mais baixos dos cinco cartões e mais próximos um do outro, indicando que, naqueles cartões, a ocorrência de respostas de distorção perceptiva se aproxima da de respostas de boa forma. Por sua vez, os valores dos respectivos desvios padrão são relativamente elevados, o que justifica o nível menor de correlação. Pode-se compreender melhor esses resultados, ao se analisar os resultados da próxima Tabela 3. Nos agrupamentos dos cartões vermelhos e coloridos, as correlações são negativas e significativas, como previsível. Esses resultados, pela sua regularidade, revestem-se de grande importância tanto quanto deixam prever que as diferenças de resultados obtidos nas versões cromática e acromática dos cartões não serão de grande monta.

Uma inspecção dos resultados constantes da Tabela 3 revela que os cartões II e IX têm médias próximas, indicando que as formas que suscitam podem assumir, em proporções próximas, a boa qualidade ou a distorção perceptiva, em ambas as versões dos cartões. São os cartões que, tal como se verificou no estudo anterior com crianças de 11/12 anos (Silva \& Ferreira, 2017), suscitam as médias mais baixas de respostas XA e médias relativamente elevadas de F-, ocorrendo mesmo, no Cartão IX, uma média de $\mathrm{F}$ - superior à de XA. 
Tabela 3

Resultados do Teste de Igualdade de Valores Médios para Amostras Emparelhadas Relativos às Respostas Dadas às Variáveis $\mathrm{XA}$ e F-nos Cartões II, III, VIII, IX, X e II+III e VIII+IX+X do Rorschach

\begin{tabular}{|c|c|c|c|c|c|c|c|c|}
\hline \multirow{2}{*}{ Cartões } & \multicolumn{2}{|c|}{$\mathrm{XA}$} & \multicolumn{2}{|c|}{ F- } & \multirow{2}{*}{$t$} & \multirow{2}{*}{$g l$} & \multirow{2}{*}{$p$} & \multirow{2}{*}{$\begin{array}{l}d^{1} \mathrm{de} \\
\text { Cohen }\end{array}$} \\
\hline & $\mathrm{M}$ & $\mathrm{DP}$ & $\mathrm{M}$ & $\mathrm{DP}$ & & & & \\
\hline II & 0,83 & 1,21 & 0,70 & 0,85 & 0,68 & 79 & 0,50 & 0,12 \\
\hline III & 1,44 & 1,65 & 0,65 & 0,73 & 3,43 & 79 & $0,00^{* *}$ & 0,62 \\
\hline VIII & 1,09 & 1,48 & 0,69 & 0,85 & 1,82 & 79 & 0,07 & 0,33 \\
\hline IX & 0,85 & 1,33 & 0,90 & 0,89 & $-0,25$ & 79 & 0,80 & 0,04 \\
\hline $\mathrm{X}$ & 1,93 & 2,02 & 1,19 & 1,07 & 2,54 & 79 & $0,01^{*}$ & 0,46 \\
\hline II+III & 2,26 & 2,14 & 1,35 & 1,16 & 3,07 & 79 & $0,00^{* *}$ & 0,53 \\
\hline VIII+IX+X & 3,86 & 3,21 & 2,78 & 1,93 & 2,27 & 79 & $0,03^{*}$ & 0,41 \\
\hline
\end{tabular}

Nota. ${ }^{*} p<.05 ;{ }^{* *} p<.05 ; .01$. $d$ de Cohen=Medida da dimensão do efeito para o Teste de igualdade de valores médios em amostras emparelhadas

Esse dado acompanha os achados geralmente reconhecidos na literatura científica, pois esses cartões consideram-se de maior dificuldade de resposta muito embora, particularmente o Cartão IX, seja aquele que, após o Cartão X, admite maior número de áreas de resposta. Isso não impede, igualmente, que, na amostra presente, seja este o único em que a média de F- (distorção perceptiva) é mais alta do que a de XA (boa forma). Esses dados levam a considerar que o Cartão IX tem características estruturais particulares que se traduzem, por um lado, pelo elevado número de áreas de resposta que suscita, e, por outro, pela fraca qualidade formal. Importa destacar, com pertinência, que, sendo o Cartão IX dotado do segundo maior número de áreas de resposta, é também um dos que suscitam menor número de respostas e maior frequência de distorções perceptivas. Daqui a afirmação da sua dificuldade, no que respeita, sobretudo, à capacidade de sugerir aos respondentes objectos com formas compatíveis com as áreas de resposta identificadas. Nos cartões III e X, o número de respostas em XA é significativamente mais elevado do que o de $\mathrm{F}$-, embora a dimensão do efeito seja pequena. No cartão VIII, tal número não chega a ser mais elevado do ponto de vista estatístico do que o de F- embora também com uma dimensão do efeito pequena as aproxima-se do nível de significância requerido, indicando que, nessa amostra, esse cartão revela-se como dotado de alguma dificuldade de resposta.

Nos dois agrupamentos, os de cartões vermelhos e coloridos, as diferenças entre as respectivas médias são estatisticamente significativas, mas a dimensão do efeito é apenas média ou pequena, respectivamente, e favoráveis à boa qualidade formal. No que respeita às médias dos cartões vermelhos II e III, o Teste de igualdade de valores médios indica elevada diferença estatisticamente significativa e uma dimensão do efeito média. No Cartão III, revela também que este não só estimula um grande número de respostas, o segundo mais alto depois do Cartão $\mathrm{X}$, como o indicam as respectivas médias, mas também que a maior parte delas é de boa qualidade formal. Por sua vez, como já foi notado, o número de respostas, no Cartão II, é o mais baixo e de fraca qualidade formal.

No que respeita ao grupo de Cartões VIII, IX e X, obtém-se diferença estatisticamente significativa entre as médias de XA e F-, embora com uma dimensão do efeito apenas pequena, o que se deve, sobretudo, ao elevado número de respostas dadas ao Cartão X. As respostas ao Cartão IX são escassas e de fraca qualidade formal e, no Cartão VIII, não revelam diferenças significativas.

Passa-se, agora, a considerar as diferenças observadas nos diversos cartões, tendo em consideração as variáveis sexo, o carácter cromático e acromático dos cartões e a influência conjunta desses dois factores, tendo em conta a Qualidade Formal das respostas. A Tabela 4 apresenta os resultados do Teste de igualdade de valores médios das respostas à variável QFo por sexo.

Esses resultados indicam a presença de uma diferença estatisticamente significativa favorável ao sexo feminino, no Cartão IX e a medida d de Cohen revela a maior dimensão do efeito nesse cartão, embora seja apenas média. Não obstante ser essa a única diferença observada estatisticamente significativa, não deixa de ser curioso que, em todos os cinco cartões, as médias no sexo feminino sejam superiores às do sexo masculino. Plausivelmente, trata-se de um indicador da maior atenção ou dedicação à tarefa revelada pelo sexo feminino ou mesmo de uma maior facilidade desse gênero em lidar com as exigências perceptivas desse Cartão IX.

A Tabela 5 apresenta os resultados do Teste de igualdade de valores médios da variável QFo nas versões cromática e acromática dos cartões. Não se verifica nenhuma diferença estatisticamente significativa entre as médias de QFo obtidas na versão cromática e acromática e a dimensão do efeito observada é sempre muito pequena, ou pequena no cartão X. Em todos os cartões, as médias na versão cromática são superiores aos da versão acromática, aspecto que abona em favor da versão padronizada. Vale a pena referir que, diferentemente do que ocorreu no estudo anterior com crianças de 11/12 anos (Silva \& 
Ferreira, 2017), no presente estudo, não ocorre a diferença estatisticamente significativa ocorrida no Cartão X, interpretada como contribuindo para aumentar o número de respostas de boa forma.

Tabela 4

Resultados do Teste de Igualdade de Valores Médios das Respostas Dadas à Variável Qfo por Sexo

\begin{tabular}{|c|c|c|c|c|c|c|c|c|c|}
\hline & \multirow{2}{*}{ Cartões } & \multicolumn{2}{|c|}{ Feminino } & \multicolumn{2}{|c|}{ Masculino } & \multirow{2}{*}{$t$} & \multirow{2}{*}{ gl } & \multirow{2}{*}{ Sig. } & \multirow{2}{*}{$\begin{array}{c}\mathrm{d} \text { de } \\
\text { Cohen }\end{array}$} \\
\hline & & M & $D P$ & M & $D P$ & & & & \\
\hline II & & 1,30 & 1,02 & 1,25 & 0,93 & 0,23 & 78 & 0,82 & 0,05 \\
\hline II & & 1,73 & 0,99 & 1,60 & 0,98 & 0,57 & 78 & 0,57 & 0,13 \\
\hline & & 1,35 & 0,77 & 1,23 & 0,66 & 0,78 & 78 & 0,44 & 0,17 \\
\hline I & & 0,95 & 0,96 & 0,50 & 0,68 & 2,42 & 78 & $0,02^{*}$ & 0,54 \\
\hline $\mathrm{x}$ & & 1,41 & 1,24 & 1,35 & 1,25 & 1,61 & 78 & 0,11 & 0,41 \\
\hline
\end{tabular}

Nota. ${ }^{*} p<0.05$

Tabela 5

Resultados do Teste de Igualdade de Valores Médios das Respostas Dadas à Variável QFo por Versão Cromática e Acromática de cada Cartão

\begin{tabular}{|c|c|c|c|c|c|c|c|c|}
\hline & \multicolumn{2}{|c|}{ Crom } & \multicolumn{2}{|c|}{ Acrom } & \multirow{2}{*}{$t$} & \multirow{2}{*}{$g l$} & \multirow{2}{*}{ Sig. } & \multirow{2}{*}{$\begin{array}{c}d \text { de } \\
\text { Cohen }\end{array}$} \\
\hline & M & $D P$ & M & $D P$ & & & & \\
\hline II & 1,35 & 1,00 & 1,20 & 0,94 & 0,69 & 78 & 0,49 & 0,16 \\
\hline II & 1,73 & 1,04 & 1,60 & 0,93 & 0,57 & 78 & 0,57 & 0,13 \\
\hline & 1,30 & 0,65 & 1,28 & 0,78 & 0,16 & 78 & 0,88 & 0,03 \\
\hline IY & 0,80 & 0,99 & 0,65 & 0,70 & 0,78 & 78 & 0,44 & 0,18 \\
\hline $\mathrm{X}$ & 1,80 & 1,32 & 1,35 & 1,17 & 1,61 & 78 & 0,11 & 0,36 \\
\hline
\end{tabular}

Um dado que merece ser destacado, por reforçar o que foi dito antes sobre o Cartão IX, é o do valor claramente mais baixo das médias de QFo em ambas as versões, médias que se acompanham de valores de desvios padrão muito elevados.

A Tabela 6 apresenta os resultados da análise de variância a dois fatores (cromático/acromático e sexo) com respeito às respostas QFo. Revela que, quando se tomam em consideração os dois factores referidos, verifica-se que um deles, o factor sexo, é o responsável exclusivo da influência expressa pela elevação acentuada da boa qualidade perceptiva das respostas, observando-se uma dimensão do efeito média.

Aqui se reencontra o efeito já antes assinalado, ao analisar os resultados do Teste de igualdade de valores médios de FQo. A Análise de Variância põe de manifesto o ponto acima salientado da maior frequência de QFo nas respostas dadas pelo sexo feminino, além da diferença significativa ocorrida no Cartão IX.

Tabela 6

Resultados da Análise da Variância a Dois Fatores (Cromático/Acromático e Sexo) das Respostas Dadas à Variável QFo

\begin{tabular}{|c|c|c|c|c|c|c|c|c|c|c|}
\hline Cartão & Factores & \multicolumn{4}{|c|}{ Estatísticas Descritivas } & $F$ & gl & Sig. & $\eta^{2} p$ & $\begin{array}{l}\text { Potência } \\
\text { do teste }\end{array}$ \\
\hline \multirow{7}{*}{$\begin{array}{l}\text { Cartão } \\
\text { IX }\end{array}$} & \multirow[b]{2}{*}{ Sexo } & \multicolumn{2}{|c|}{ Feminino } & \multicolumn{2}{|c|}{ Masculino } & & & & & \\
\hline & & \multicolumn{2}{|c|}{$\begin{array}{c}0,95 \\
(0,96)\end{array}$} & \multicolumn{2}{|c|}{$\begin{array}{c}0,50 \\
(0,68)\end{array}$} & 6,09 & 1 & $0,02^{*}$ & 0,07 & 0.68 \\
\hline & \multirow[b]{2}{*}{ Crom/Acrom } & \multicolumn{2}{|c|}{ Cromático } & \multicolumn{2}{|c|}{ Acromático } & & & & & \\
\hline & & \multicolumn{2}{|c|}{$\begin{array}{c}0,80 \\
(0,99)\end{array}$} & \multicolumn{2}{|c|}{$\begin{array}{c}0,65 \\
(0,70)\end{array}$} & 0,67 & 1 & 0,42 & 0,00 & 0,13 \\
\hline & \multirow{3}{*}{$\begin{array}{l}\text { Sexo* Crom/ } \\
\text { acrom }\end{array}$} & \multicolumn{2}{|c|}{ Cromático } & \multicolumn{2}{|c|}{ Acromático } & \multirow{3}{*}{3,65} & \multirow{3}{*}{1} & \multirow{3}{*}{0,06} & \multirow{3}{*}{0,05} & \multirow{3}{*}{0,47} \\
\hline & & $\mathrm{F}$ & $\mathrm{M}$ & $\mathrm{F}$ & $\mathrm{M}$ & & & & & \\
\hline & & $\begin{array}{c}1.20 \\
(1.15)\end{array}$ & $\begin{array}{c}0,40 \\
(0,60)\end{array}$ & $\begin{array}{c}0,70 \\
(0,66)\end{array}$ & $\begin{array}{c}0,60 \\
(0,75)\end{array}$ & & & & & \\
\hline
\end{tabular}

Nota. ${ }^{*} p<.05$ 
A Tabela 7 apresenta os resultados do Teste de igualdade de valores médios da variável QF- por sexo. Não se encontra nenhuma diferença significativa entre o número médio de respostas em nenhum dos cinco cartões em estudo, sendo a dimensão do efeito sempre muito pequena ou pequena (cartão X). Não deixa de ser curioso observar que, enquanto o sexo feminino apresenta, nos cartões vermelhos II e III, médias ligeiramente superiores às do sexo masculino, este apresenta valores mais elevados do que o feminino nos cartões coloridos, observando-se mesmo um pequeno efeito no Cartão X. Vale a pena registar a elevação dos desvios padrão em todos os casos, indicadores da grande dispersão dos resultados.

Tabela 7

Resultados do Teste de Igualdade de Valores Médios das Respostas Dadas à Variável QF- por Sexo

\begin{tabular}{|c|c|c|c|c|c|c|c|c|c|}
\hline & \multirow{2}{*}{ Cartões } & \multicolumn{2}{|c|}{ Feminino } & \multicolumn{2}{|c|}{ Masculino } & \multirow{2}{*}{$t$} & \multirow{2}{*}{$g l$} & \multirow{2}{*}{ Sig. } & \multirow{2}{*}{$\begin{array}{c}d \text { de } \\
\text { Cohen }\end{array}$} \\
\hline & & $M$ & $D P$ & $M$ & $\mathrm{DP}$ & & & & \\
\hline I] & & 0,75 & 0,95 & 0,65 & 0,74 & 0,53 & 78 & 0,60 & 0,12 \\
\hline Il & & 0,68 & 0,80 & 0,63 & 0,67 & 0,30 & 78 & 0,76 & 0,07 \\
\hline & & 0,63 & 0,90 & 0,75 & 0,81 & $-0,66$ & 78 & 0,52 & 0,14 \\
\hline I. & & 0,80 & 0,85 & 1,00 & 0,93 & $-1,00$ & 78 & 0,32 & 0,23 \\
\hline$x$ & & 0,98 & 0,92 & 1,40 & 1,17 & $-1,80$ & 73,81 & 0,08 & 0,40 \\
\hline
\end{tabular}

A Tabela 8 apresenta os resultados do Teste de Igualdade de valores médios da variável $\mathrm{QF}$ - por versão cromática e acromática de cada cartão. Como pode ver-se, não ocorrem quaisquer diferenças significativas em nenhum dos cartões estudados e a dimensão do efeito é sempre muito pequena. Esse dado reveste-se de importância, pois, em relação ao estudo anterior com crianças de 11/12 anos (Silva \& Ferreira, 2017), desaparece a diferença significativa que ali ocorre no Cartão III, reveladora, como se referiu inicialmente, de que a cor vermelha era responsável pelo aumento das respostas de distorção perceptiva naquele cartão. Este aparenta ser um novo indicador de desenvolvimento que pode considerar-se em consonância com o facto antes referido de a cor determinar o aumento da produção de respostas aos cartões coloridos a partir dos 15/16 anos. Se se pôde afirmar, em trabalho anterior (Silva \& Marques, 2008), que, durante o período etário dos 5/6 aos 11/12 anos, a cor parece ter um efeito inibitório na produção de respostas bem como determinar o aumento significativo da distorção perceptiva no Cartão III, é razoável pensar-se que, a par da ausência da inibição e aquisição da capacidade de aumentar a produção de respostas ocorra, igualmente, a ausência de aumento significativo da referida distorção perceptiva. Este constitui um dado que invalida muito claramente a nossa hipótese inicial da influência da cor na qualidade formal das respostas, designadamente na distorção perceptiva após os 15/16 anos.

Tabela 8

Resultados do Teste de Igualdade de Valores Médios das Respostas Dadas à Variável QF_por Versão Cromática e Acromática de cada Cartão

\begin{tabular}{|c|c|c|c|c|c|c|c|c|c|}
\hline & \multirow{2}{*}{ Cartões } & \multicolumn{2}{|c|}{ Cromático } & \multicolumn{2}{|c|}{ Acromático } & \multirow{2}{*}{$t$} & \multirow{2}{*}{ gl } & \multirow{2}{*}{ Sig. } & \multirow{2}{*}{$\begin{array}{c}\mathrm{d} d \mathrm{de} \\
\text { Cohen }\end{array}$} \\
\hline & & $M$ & $D P$ & M & $\mathrm{DP}$ & & & & \\
\hline II & & 0,73 & 0,82 & 0,68 & 0,89 & 0,26 & 78 & 0,79 & 0,06 \\
\hline Il & & 0,63 & 0,74 & 0,68 & 0,73 & $-0,30$ & 78 & 0,76 & 0,07 \\
\hline & & 0,68 & 0,83 & 0,70 & 0,88 & $-0,13$ & 78 & 0,90 & 0,02 \\
\hline I. & & 0,83 & 0,84 & 0,94 & 0,95 & $-0,75$ & 78 & 0,46 & 0,12 \\
\hline$x$ & & 1,20 & 1,07 & 1,18 & 1,08 & 0,10 & 78 & 0,92 & 0,02 \\
\hline
\end{tabular}

A Tabela 9 apresenta os resultados do Teste de Igualdade de valores médios, das respostas QFu dadas por sexo. Como se vê, não ocorrem diferenças significativas e as dimensões do efeito são sempre muito pequenas em todos cartões. Poderíamos apenas dizer, a partir dos resultados obtidos, que o factor sexo não tem efeito determinante na maior ou menor ocorrência das respostas codificadas QFu.

A Tabela 10 contém os resultados do Teste de valores médios das respostas $\mathrm{QFu}$ nas versões cromática e acromática dos cartões em estudo. Esses resultados mostram que, com excepção do Cartão III, em todos 
os restantes a frequência de QFu tende a ser mais elevada na versão cromática do que na acromática, atingindo diferença estatisticamente significativa e uma dimensão do efeito pequena no Cartão X. No Cartão IX, embora não se observe diferença significativa entre o número médio de respostas dadas também se regista uma dimensão do efeito pequena. Esses dados permitem reconhecer a importância da cor na ocorrência desse tipo de respostas e o quanto contribui para o enriquecimento da qualidade perceptiva e para a propriedade diferenciadora das respostas, particularmente no Cartão X.

Tabela 9

Resultados do Teste de Igualdade de Valores Médios das Respostas Dadas à Variável QFu por Sexo

\begin{tabular}{|c|c|c|c|c|c|c|c|c|}
\hline \multirow{2}{*}{ Cartões } & \multicolumn{2}{|c|}{ Feminino } & \multicolumn{2}{|c|}{ Masculino } & \multirow{2}{*}{$t$} & \multirow{2}{*}{ gl } & \multirow{2}{*}{ Sig. } & \multirow{2}{*}{$\begin{array}{c}d \text { de } \\
\text { Cohen }\end{array}$} \\
\hline & M & DP & $M$ & $D P$ & & & & \\
\hline II & 0,77 & 0,64 & 0,53 & 0,64 & 0,81 & 78 & 0,42 & 0,38 \\
\hline III & 0,58 & 0,78 & 0,83 & 0,87 & $-1,35$ & 78 & 0,18 & 0,31 \\
\hline VIII & 0,78 & 0,97 & 0,65 & 0,80 & 0,63 & 78 & 0,53 & 0,15 \\
\hline IX & 0,93 & 0,66 & 0,83 & 0,84 & 0,59 & 78 & 0,56 & 0,13 \\
\hline$X$ & 1,35 & 1,05 & 1,10 & 0,90 & 1,14 & 78 & 0,26 & 0,26 \\
\hline
\end{tabular}

Tabela 10

Resultados do Teste de Igualdade de Valores Médios das Respostas Dadas à Variável QFu por Versão Cromática e Acromática de cada Cartão

\begin{tabular}{|c|c|c|c|c|c|c|c|c|}
\hline \multirow{2}{*}{ Cartões } & \multicolumn{2}{|c|}{ Crom } & \multicolumn{2}{|c|}{ Acrom } & \multirow{2}{*}{$t$} & \multirow{2}{*}{ gl } & \multirow{2}{*}{ Sig. } & \multirow{2}{*}{$\begin{array}{c}d \text { de } \\
\text { Cohen }\end{array}$} \\
\hline & $M$ & $D P$ & M & $D P$ & & & & \\
\hline II & 0,68 & 0,73 & 0,50 & 0,64 & 1,14 & 78 & 0,26 & 0,26 \\
\hline III & 0,63 & 0,77 & 0,78 & 0,89 & $-0,80$ & 78 & 0,42 & 0,18 \\
\hline VIII & 0,85 & 0,95 & 0,58 & 0,81 & 1,39 & 78 & 0,17 & 0,15 \\
\hline IX & 0,98 & 0,83 & 0,78 & 0,66 & 1,19 & 78 & 0,24 & 0,27 \\
\hline $\mathrm{X}$ & 1,45 & 0,99 & 1,00 & 0,93 & 2,10 & 78 & $0,04^{*}$ & 0,47 \\
\hline
\end{tabular}

Nota. ${ }^{*} p<.05$

A Tabela 11 contém os resultados do Teste de igualdade de valores médios em das respostas XA, isto é, respostas com boa forma, por sexo. A sua inspecção revela a ocorrência de uma diferença estatisticamente significativa e uma pequena dimensão do efeito, no cartão IX, favorável ao sexo feminino. Essa diferença confirma a observada na comparação entre médias de QFo por sexo destacada na Tabela 4. Note-se que, embora, no Cartão X, não se registem diferenças significativas, observa-se também uma pequena dimensão do efeito, no mesmo sentido, favorável ao sexo feminino. Esse dado permite considerar que, nos cartões IX e X, onde a frequência de distorção perceptiva é mais elevada, particularmente no sexo masculino, como se pode observar na Tabela 7, encontramos a diferença mais acentuada entre o número médio de respostas de boa qualidade formal entre sexos, favoráveis ao sexo feminino. Esse facto permite salientar que, nessa amostra, o sexo feminino logra encarar a particularidade estrutural do Cartão IX, atrás assinalada, e a dificuldade do Cartão $\mathrm{X}$, de maneira mais eficiente e adequada do que o sexo masculino.

Tabela 11

Resultados do Teste de Igualdade de Valores Médios das Respostas Dadas à Variável XA por Sexo

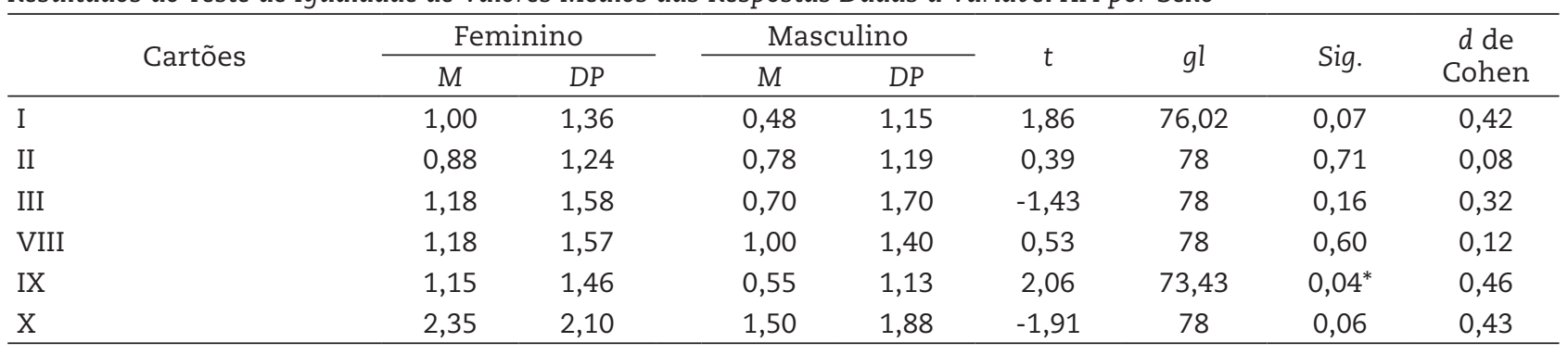

Nota. ${ }^{*} p<.05$ 
A Tabela 12 apresenta os resultados do Teste de igualdade de valores médios das respostas à variável XA tendo em conta as versões cromática e acromática dos cartões. O exame desses resultados revela, antes de mais, que, com excepção do cartão III, em todos os demais se obtêm médias mais baixas na versão acromática do que na cromática, um dado bastante favorável à qualidade do Rorschach como instrumento de avaliação. Com efeito, se se verificasse a predominância de más formas na versão cromática, muito provavelmente o teste apresentaria ou seria considerado como de grande dificuldade de resposta, aspecto naturalmente com efeito negativo nos seus objectivos. Um outro dado importante é o de que, no Cartão X, a média de XA na versão cromática é significativamente mais elevada do que a da versão acromática e se observa uma dimensão do efeito média. Tínhamos assinalado, na análise dos resultados da variável anterior, que, no Cartão X, se encontrava uma dimensão do efeito pequena embora não se revele significativo, aspecto que é, agora, efectivamente alcançado.

Tabela 12

Resultados do Teste de Igualdade de Valores Médios das Respostas Dadas à Variável XA por Versão Cromática e Acromática de cada Cartão

\begin{tabular}{|c|c|c|c|c|c|c|c|c|c|}
\hline & \multirow{2}{*}{ Cartões } & \multicolumn{2}{|c|}{ Crom } & \multicolumn{2}{|c|}{ Acrom } & \multirow{2}{*}{$t$} & \multirow{2}{*}{ gl } & \multirow{2}{*}{ Sig. } & \multirow{2}{*}{$\begin{array}{c}\text { d de } \\
\text { Cohen }\end{array}$} \\
\hline & & M & $D P$ & $M$ & DP & & & & \\
\hline I & & 0,83 & 1,39 & 0,65 & 1,17 & 0,61 & 78 & 0,54 & 0,14 \\
\hline I & & 1,03 & 1,33 & 0,63 & 1,06 & 1,48 & 74,15 & 0,14 & 0,33 \\
\hline & & 1,40 & 1,69 & 1,48 & 1,63 & $-0,20$ & 78 & 0,84 & 0,05 \\
\hline & & 1,33 & 1,61 & 0,85 & 1,31 & 1,45 & 74,98 & 0,15 & 0,33 \\
\hline I & & 1,03 & 1,54 & 0,68 & 1,07 & 1,18 & 69,49 & 0,24 & 0,27 \\
\hline$\underline{Y}$ & & 2,50 & 2,20 & 1,35 & 1,67 & 2,64 & 72,86 & $0,01^{*}$ & 0,59 \\
\hline
\end{tabular}

Nota. ${ }^{*} p=.01$

Vale a pena destacar, a partir da análise das tabelas anteriores referentes ao comportamento dos valores médios das variáveis FQo e FQu, componentes da variável XA, que, no caso da primeira, as médias obtidas são sempre superiores na versão cromática, mas nem sempre com uma diferença nítida. Por outro lado, na segunda, FQu, tais diferenças são claramente superiores, com excepção do Cartão III cuja média é mais elevada na versão acromática. Queremos, desse modo, salientar a importância da variável QFu na determinação da diferença significativa, favorável à versão cromática, alcançada no Cartão X.

A Tabela 13 contém os resultados da Análise de Variância a dois factores - cromático/acromático e sexo relativos às respostas XA. Eles indicam a existência efectiva da influência do factor sexo na diferença entre valores médios das respostas, embora apenas com um efeito de pequena dimensão, no Cartão $\mathrm{IX}$, mas sem repercussão na distribuição dessas respostas em função do carácter cromático ou acromático dos cartões.

Tabela 13

Resultados da Análise da Variância a Dois Fatores (Cromático/Acromático e Sexo) das Respostas Dadas à Variável XA

\begin{tabular}{|c|c|c|c|c|c|c|c|c|c|c|}
\hline Cartões & Fatores & \multicolumn{4}{|c|}{ Estatísticas descritivas } & $F$ & $g l$ & Sig. & $\eta^{2} p$ & $\begin{array}{l}\text { Potência } \\
\text { do teste }\end{array}$ \\
\hline \multirow{7}{*}{$\begin{array}{l}\text { Cartão } \\
\text { IX }\end{array}$} & \multirow[b]{2}{*}{ Sexo } & \multicolumn{2}{|c|}{ Masculino } & \multicolumn{2}{|c|}{ Feminino } & & & & & \\
\hline & & \multicolumn{2}{|c|}{$\begin{array}{c}1,15 \\
(1,46)\end{array}$} & \multicolumn{2}{|c|}{$\begin{array}{c}0,55 \\
(1,13)\end{array}$} & 4,21 & 1 & $0,04^{*}$ & 0,05 & 0,53 \\
\hline & & \multicolumn{2}{|c|}{ Cromático } & \multicolumn{2}{|c|}{ Acromático } & & & & & \\
\hline & $\begin{array}{l}\text { Crom/ } \\
\text { Acrom }\end{array}$ & \multicolumn{2}{|c|}{$\begin{array}{c}1,03 \\
(1,54)\end{array}$} & \multicolumn{2}{|c|}{$\begin{array}{c}, 68 \\
(1,07)\end{array}$} & 1,43 & 1 & 0,24 & 0,02 & 0,24 \\
\hline & \multirow{3}{*}{$\begin{array}{c}\text { Sexo*Crom/ } \\
\text { Acrom }\end{array}$} & \multicolumn{2}{|c|}{ Crom } & \multicolumn{2}{|c|}{ Acrom } & \multirow{3}{*}{0,26} & \multirow{3}{*}{1} & \multirow{3}{*}{0,61} & \multirow{3}{*}{0,00} & \multirow{3}{*}{0,08} \\
\hline & & $\mathrm{F}$ & $\mathrm{M}$ & $\mathrm{F}$ & $\mathrm{M}$ & & & & & \\
\hline & & $\begin{array}{c}1,40 \\
(1,70)\end{array}$ & $\begin{array}{c}0,65 \\
(1,31)\end{array}$ & $\begin{array}{c}0,90 \\
(1,17)\end{array}$ & $\begin{array}{l}0,45 \\
(, 95)\end{array}$ & & & & & \\
\hline
\end{tabular}

Nota. ${ }^{*} p<.05$ 


\section{Discussão}

Ao iniciar esta comunicação, referiu-se que os estudos sobre o efeito da cor no aumento da produção de respostas aos cartões coloridos (Silva, 2002, 2009; Silva \& Ferreira, 2011, 2014) mostraram que tal efeito só se estabelecia, no período etário dos 15/16 anos. Por outro lado, um outro estudo, em que se procurou avaliar se a cor poderia ser responsável pela elevada ocorrência de respostas de distorção perceptiva nos cartões com cor, revelou, com um grupo de 11/12 anos, que tal acontecia apenas no Cartão III (Silva \& Ferreira, 2017) cujo valor médio de distorções era significativamente mais elevado, na versão cromática do que na versão acromática. Considerada a relação estabelecida entre cor e produção de respostas e cor e qualidade formal das respostas, fomos levados a repetir o estudo efectuado com crianças, agora com uma amostra de idade igual ou superior a 15/16 anos cujos resultados acabamosde apresentar.

Estes permitem, com efeito, detectar alguns indicadores do efeito do desenvolvimento após os 11/12 anos. Começando por tomar a variável "boa qualidade formal ou QFo", verificamos que, no grupo dos jovens, as médias obtidas são mais elevadas na versão cromática ou padronizada dos cinco cartões do que na versão acromática, embora tais diferenças não sejam estatisticamente significativas; no grupo das crianças, tais valores são mais elevados nos Cartões II, VIII e X, significativamente neste último, mas não no Cartão III nem no IX, cujo valor é igual ao da versão acromática. Temos, pois, que, nos jovens, a cor favorece a boa qualidade formal, em comparação com o que se observa nas crianças.

Passando à variável $\mathrm{FQu}$, código usado para as boas formas de ocorrência rara, verifica-se que o grupo dos jovens obtém médias mais altas na versão cromática do que na acromática, nos Cartões II, VIII, IX e X, ao passo que, no grupo das crianças, tal acontece apenas em VIII, IX e X. Saliente-se que, no Cartão X e em ambos os grupos, a média obtida na versão cromática é significativamente mais alta do que a da versão acromática. Seja com for, regista-se a maior facilidade, no grupo dos jovens do que no das crianças, em admitir a cor na formulação de respostas, particularmente face aos cartões vermelhos, nos quais o número dessas respostas, nas crianças, é mais elevado na versão acromática do que na cromática.

Embora a variável XA represente a soma das variáveis QFo e QFu, referimos também os resultados obtidos para salientar um dado digno de nota. No grupo dos jovens, as médias mais elevadas na versão cromática do que na acromática ocorrem nos Cartões II, VIII, IX e X, ao passo que, no grupo das crianças, eles ocorrem, de novo, apenas em VIII, IX e X, confirmando o antes observado.

Finalmente, vejamos o que se passa com a variável QF-. No grupo de jovens, as médias mais altas obtidas na versão cromática ocorrem nos Cartões II e $\mathrm{X}$; no grupo das crianças, ocorrem nos Cartões II, III e X, com diferença estatisticamente significativa no Cartão III, o que permite identificar este Cartão como aquele em que a cor vermelha suscita um número particularmente elevado de distorções perceptivas. Aqui se insere o dado digno de nota há pouco referido. Se considerarmos o comportamento das médias QF- obtidas no Cartão III, em ambos os grupos, vemos que de significativamente mais alta, na versão cromática, no grupo das crianças, tal média passa a ser apenas menos alta, no grupo dos jovens, mas mantém-se menos elevada na versão cromática do que na acromática, não apenas no Cartão III mas também nos Cartões VIII e IX. Isso significa que, mesmo na idade mais avançada dos jovens, persiste alguma dificuldade em dar respostas de boa qualidade formal, em três dos cinco cartões com cor. Isto verifica-se, sobretudo no sexo masculino, como decorre da análise anterior dos valores médios das respostas QFo por sexo. Com efeito, esta análise revela uma diferença significativa no Cartão IX, favorável ao sexo feminino e médias de QFo superiores às do sexo masculino nos cinco cartões estudados. Este constitui um dado interessante que importa confirmar em novos estudos.

Pode, pois, considerar-se que existe uma mudança no efeito da cor sobre a qualidade formal das respostas aos cartões com cor do Rorschach, entre os 11/12 anos e os anos 15/16 e 21 anos, que se expressa pela menor frequência de distorções perceptivas e maior frequência de boas formas na versão cromática ou padronizada dos cartões, no grupo dos jovens em relação ao grupo de crianças.

\section{Conclusão}

Face à hipótese inicial de que a cor seria responsável pela maior frequência de respostas QF-, os resultados obtidos permitem admitir que tal hipótese é parcialmente confirmada em crianças de 11/12 anos e eventualmente até aos 14 , mas que tal não se verifica a partir dos 15/16 anos, período em que a cor deixa de constituir obstáculo à solução do problema que constitui reconhecer um objecto numa mancha colorida. Com efeito, mediante o desenvolvimento perceptivo, que se opera ao longo dos primeiros anos de acesso às operações formais, a cor passa a ser uma característica do real, facilitadora do reconhecimento de formas, donde o aumento da sua produção sem prejuízo da qualidade formal.

Tanto quanto verificámos, não encontrámos, na bibliografia consultada, indícios de estudos dentro desta temática, cor e qualidade formal no Rorschach. Seria vantajoso que outros estudos confirmassem os resultados aqui propostos. 


\section{Referências}

Exner, J. E. (2003). The Rorschach A Comprehensive System Basic Foundations and Principles of Interpretation Vol. 1 (4th edition). New Jersey, John Wiley \& Sons.

Exner, J. E., \& Erdberg, P. (2005). The Rorschach A Comprehensive System. Volume 2 (3rd edition). New Jersey, John Wiley \& Sons.

Pires, A. A. (2007). Rorschach Comprehensive System Data for a Sample of 309 Adult Nonpatients from Portugal. Journal of Personality Assessment, 89, Supplement (S1), S124-S130.

Silva, D. R. (2002). The effect of color on productivity on card X of the Rorschach. Rorschachiana, 25(1), 123-138.doi: 10.1027/11925604.25.1.123

Silva, D. R., \& Marques, L. G. (2008). L'effet de la couleur sur la production de réponses au Rorschach chez des enfants non consultants: Une étude génétique. Psychologie Clinique et Projective, 14, 221-233.doi: 10.3917/pcp.014.0221

Silva, D. R., \& Marques, L. G. (2009). Função da cor na produção de respostas ao Rorschach até ao final da adolescência. Revista IberoAmericana de Diagnóstico y Evaluación le Avaliação Psicológica, 27(2), 149-165.

Silva, D. R., \& Ferreira, A. S. (2011). Cor e produção de respostas ao Rorschach: Um estudo transcultural. Paidéia, (Ribeirão Preto), 21(49), 149-156.doi: 10.1590/S0103-863X2011000200002

Silva, D. R., \& Ferreira, A. S. (2014). The effect of color on the production of responses to Rorschach Cards VIII, IX, and X in age groups of 11-2 and 15-16 years. Journal of Personality Assessment, 96(4), 1-6. doi: 10.1080/00223891.2013.876426

Silva, D. R., \& Ferreira, A. S. (2017). Cor e qualidade formal no método de Rorschach em um Grupo de Crianças de 11/12 Anos de Idade. Avaliação Psicológica, 16(1), 6-10.

\section{Sobre os autores}

Danilo Rodrigues Silva é Professor Catedrático aposentado da Faculdade de Psicologia da Universidade de Lisboa, na área de Avaliação Psicológica, sendo Doutorado em Psicologia pela mesma universidade. Foi Presidente da Associação Iberoamericana de Diagnóstico e Avaliação Psicológica e membro correspondente da Academia das Ciências de Lisboa.

Ana Sousa Ferreira é Doutorada e Mestre em Matemática Aplicada - Estatística (Univ. Nova de Lisboa e Univ. de Lisboa). Professora Auxiliar da Faculdade de Psicologia, Universidade de Lisboa e Investigadora na Business Research Unit - (BRU) - Instituto Universitário de Lisboa. 